\title{
Cardiac Remodelling in Patients Undergoing in-Centre Nocturnal Haemodialysis: Results from the MIDNIGHT Study, a Non-Randomized Controlled Trial
}

\author{
Matthew P.M. Graham-Brown ${ }^{a-c}$ Darren R. Churchward ${ }^{b, c}$ Katherine L. Hullb, c \\ Rob Preston ${ }^{b}$ Warren P. Pickering ${ }^{b}$ Helen C. Eboralld Gerry P. McCann ${ }^{e}$ \\ James O. Burton ${ }^{\mathrm{a}-\mathrm{c}, \mathrm{e}}$ \\ ${ }^{a}$ National Centre for Sport and Exercise Medicine, Loughborough, bohn Walls Renal Unit, University Hospitals of \\ Leicester NHS Trust, ' Department of Infection Immunity and Inflammation, University of Leicester, ${ }^{\mathrm{d}}$ Department of \\ Health Sciences, University of Leicester, and ${ }^{\mathrm{e}}$ Department of Cardiovascular Sciences, University of Leicester and \\ NIHR Leicester Biomedical Research Centre, Glenfield Hospital, Leicester, UK
}

\section{Keywords}

Nocturnal haemodialysis · Cardiac MRI · Left ventricular mass · Native T1 · Myocardial fibrosis

\begin{abstract}
Evidence suggests extended-hours haemodialysis (HD) may improve cardiovascular, medical and quality-of-life outcomes. In-centre nocturnal haemodialysis (INHD) is an established but underutilized method of providing extendedhours treatment. This 6-month, non-randomized controlled trial (ISRCTN16672784) recruited 13 INHD patients and 12 control patients on conventional HD. The effects of treatment on left ventricular (LV) structure, function and myocardial fibrosis were assessed using cardiac magnetic resonance imaging and native T1 mapping. Quality-of-life and clinical measures were also collected. INHD led to significant reductions in LV mass (-14.75 vs. $+6.54 \mathrm{~g} ; p=0.02)$, global T1 $(-30.62$ vs. $0.4 \mathrm{~ms} ; p=0.05)$ and non-septal native T1 values ( -30.93 vs. $8.96 \mathrm{~ms} ; p=0.02)$ over time. There were also significant improvements in serum phosphate $(-0.39$ vs. +0.02 $\mathrm{mmol} / \mathrm{L} ; p=0.03$ ) and reductions in ultrafiltration rates
\end{abstract}

\section{KARGER}

(c) 2017 S. Karger AG, Basel

E-Mail karger@karger.com

www.karger.com/bpu
$(-2.32$ vs. $+0.70 \mathrm{~mL} / \mathrm{h} / \mathrm{kg} p=0.05)$ between INHD and controls. Six-months of INHD was associated with favourable LV remodelling and reduced myocardial fibrosis compared to patients on conventional haemodialysis.

C) 2017 S. Karger AG, Basel

\section{Introduction}

The morbidity and mortality for maintenance haemodialysis (HD) patients is high [1], with $40 \%$ of patients dying from cardiovascular disease [2]. Cardiovascular disease in HD patients is driven by different risk factors from the general population, including: chronic inflammation; uraemia; stresses of HD; large-volume ultrafiltration; endothelial dysfunction; increased arterial stiffness and autonomic instability [3-6]. The combination of these insults results in maladaptive cardiac remodelling that leads to left ventricular (LV) hypertrophy, dilatation

M.P.M.G.-B. and D.R.C. first authors. 
and myocardial fibrosis, commonly termed uraemic cardiomyopathy [7]. Modification of traditional risk factors has not improved outcomes for HD patients as they have for patients in the general population $[8,9]$.

The stresses of conventional HD (CHD) of $4 \mathrm{~h}$ HD 3 times per week are known to adversely impact the cardiovascular system [10-12] and there is evidence that more frequent [13] and extended duration HD [14, 15] improve cardiovascular outcomes for patients. While homebased HD provides extended or more frequent HD for some patients, there are barriers (patient-related and physician-related) to many undertaking home-HD programs; in-centre nocturnal haemodialysis (INHD) is an underutilized method of delivering extended HD. Evidence suggests INHD programs are feasible [16], may improve a number of clinical endpoints [15, 17-21], reduce LV mass [22] and even improve survival [23].

Cardiac MRI (CMR) is the gold-standard for the assessment of LV structure (LV) and function. Measurement of LV dimensions is reproducible in HD patients $[24,25]$ and the graded relationship between LV mass and cardiovascular risk [26] underlines the importance of accurately quantifying LV structure in clinical trials involving HD patients. The multi-parametric nature of CMR means it can give detailed information about myocardial tissue characterization, particularly myocardial fibrosis, without the need for gadolinium-based contrast agents, which are contraindicated in patients with advanced renal disease [27-29]. Native T1 mapping is a novel, noncontrast CMR measure that correlates well with histological levels of myocardial fibrosis in patients with aortic stenosis. Our group and others have shown it is significantly raised in HD patients compared to controls [28, 29] and its outstanding reproducibility makes it an attractive biomarker for the assessment of myocardial fibrosis in dialysis patients [27].

In this feasibility study [30], we investigated the effects of 6-months extended duration INHD on cardiac structure, function and LV remodelling in prevalent HD patients.

\section{Materials and Methods}

The MIDNIGHT study (ISRCTN16672784) was approved by the National Research and Ethics committee (reference: 15/ EM/0268) and sponsored by the University of Leicester. Institutional approval was provided by University Hospitals of Leicester NHS Trust (UHL11434). Informed consent was obtained from participants.

Full details of materials and methods are as previously published [30]. This non-randomized controlled trial compared the effects of a 6-month program of INHD on cardiac structure and function in prevalent HD patients. Twelve patients were recruited who had electively switched from CHD to INHD. A control group was recruited from patients that remained on standard care. Attempts were made to match patients 1:1 for age, gender, dialysis vintage and co-morbidities. Routine clinical and demographic information was collected for all patients including haematological and biochemical parameters. Study methodology is as previously described including inclusion and exclusion criteria [30]. Outcome measures included CMR, bioimpedance spectroscopy, and the quality-of-life questionnaires: SF-12, POS-S Renal, with outcome measures repeated at 6 months.

\section{Administration of Dialysis Therapies}

Patients undertaking INHD dialyzed 3 times per week, overnight as previously described [16]. Initial dialysis time was $300 \mathrm{~min}$, increasing up to a maximum of $480 \mathrm{~min}$ as tolerated throughout the 6-month period of intervention. The control group dialyzed for 240 min 3 times per week and routine care did not change. Blood and dialysate flow rates and measures of dialysis adequacy were recorded prospectively. All aspects of HD patient care were managed according to standards set by the UK Renal Association [31].

\section{CMR Protocol and Analysis}

Patients were imaged on a 3 Tesla CMR platform (Skyra, Siemens Medical Imaging, Erlangen, Germany) using an 18-channel phased-array anterior coil. CMR protocols for acquiring cine imaging and native T1 maps were as previously described [27, 32], and conformed to internationally recognized standards [33].

CMR scans were analyzed using the software package CMR [42] (Circle Cardiovascular Imaging, Calgary, Alberta, Canada) and were analyzed offline by a single blinded observer. LV volumes and mass, native T1 and myocardial strain (global circumferential strain [GCS] and global longitudinal strain [GLS]) were quantified as previously described $[27,34]$. Aortic PWV was acquired and assessed using the software JIM version 6 (Xinapse software, UK) as previously described [35].

\section{Blood Pressure}

Blood pressure data, pre- and post-HD, were collected from patients' medical records. An average of 12 recordings (4-week in total) prior to start of INHD (intervention group) or prior to baseline CMR (control group) was calculated. Similarly, 4-week averages were calculated prior to follow-up CMR in both groups.

\section{Statistical Analysis}

Statistical analysis was undertaken using SPSS-24 software (Statistical Package for the Social Sciences, Chicago, IL, USA). Normality was assessed using the Shapiro-Wilk test, histograms and Q-Q plots. Parametric data are expressed as mean \pm SD and non-parametric data are expressed as median (interquartile range). Chi-square tests were used to assess for differences between categorical variables and are expressed as "count" (\%). A mixed model 2-way analysis of variance was used to assess for differences between INHD and CHD patients at baseline and study completion; multiple comparisons were adjusted using the Sidak's method. Within-group differences are expressed as mean difference and 95\% CIs. Changes in outcome measures were correlated using Pearson's and Spearman's rank analyses for normally and nonnormally distributed data respectively. 
Fig. 1. Study recruitment, flow and patient withdrawal. Flow diagram showing patient flow through the study. INHD, in-centre nocturnal haemodialysis; $\mathrm{CHD}$, conventional haemodialysis; MRI, magnetic resonance imaging.
Patients approached for INHD arm $(n=19)$

Matched controls approached for CHD arm $(n=12)$

INHD patients not providing consent $(n=6)$

INHD patients enrolled in study $(n=13)$

Matched CHD patients enrolled in study $(n=12)$

NHD loss to final follow-up:

Transplanted $(n=2)$

Consent withdrawn $(n=1)$

INHD patients included in final analysis $(n=10)$

N.B. MRI analysis ( $n=8 ; 2$ patients no follow-up scans)

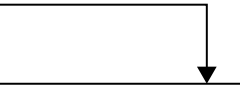

CHD group loss to final follow-up Consent withdrawn $(n=1)$

CHD patients included in final analysis $(n=11)$

N.B. MRI analysis ( $n=9$; no follow-up scan $n=1$, excluded due to regimen change $n=1$ )

\section{Results}

Twenty-five patients were enrolled between July 2015 and February 2016. Figure 1 shows patient flow through the study. Patient demographics are shown in Table 1. There were no significant differences in medication use during the study (data not shown). There were 2 dropouts from the INHD group following baseline scan due to transplantation; 1 patient withdrew consent after baseline assessment. Two further patients in the INHD group declined follow-up CMR scan. Two patients from the CHD group dropped out of the study after completing the baseline study visit. A further patient from the CHD group was excluded from final analysis due to a change in HD regimen. Eight patients who completed the 6-month INHD program and 9 control patients were included in the final CMR analysis and 10 INHD patients and $11 \mathrm{CHD}$ patients were included for final analysis of non-CMR parameters.

\section{Clinical and Quality-of-Life Parameters}

Baseline and follow-up results for clinical biochemistry, haematology, blood pressure, details of HD prescription, dialysis adequacy, bioimpedance spectroscopy and quality-of-life assessments are shown in Table 2. There were significant improvements in serum phosphate levels (mean difference $[95 \% \mathrm{CIs}] \mathrm{INHD}=-0.39 \mathrm{mmol} / \mathrm{L}[-0.70$ to -0.07$], \mathrm{CHD}=0.02 \mathrm{mmol} / \mathrm{L}$ [ -0.26 to 0.31$], p=0.03$ ) and reductions in ultrafiltration rates (mean difference [95\% CIs] INHD $=-2.32 \mathrm{~mL} / \mathrm{h} / \mathrm{kg}[-3.74$ to -0.91$]$, $\mathrm{CHD}=-0.70 \mathrm{~mL} / \mathrm{h} / \mathrm{kg}$ [ -1.98 to 0.57$], p=0.05$ ) between INHD and control patients over time. Post hoc analysis showed the differences over time in serum phosphate and ultrafiltration rates were significant only for patients in the INHD group ( $p=0.02$ and $p<0.01$, respectively).

\section{$L V$ Volumes and Mass}

Changes in LVM and volumes are shown in Table 3. There was a significant reduction in LVM between INHD and CHD groups over time (mean difference [95\% CI's] $\mathrm{INHD}=-14.75 \mathrm{~g}[-29.62$ to 0.12$], \mathrm{CHD}=6.54 \mathrm{~g}[-7.48$ to $20.57], p=0.02)$. There was also a significant reduction in $\mathrm{LVM} / \mathrm{LV}$ end-diastolic volume (LVEDV) in the INHD patients compared to controls (mean difference [95\% CIs] INHD $=-0.07 \mathrm{~g} / \mathrm{mL}[-0.15$ to 0$], \mathrm{CHD}=0.06 \mathrm{~g} / \mathrm{mL}[-0.01$ to 0.13 ], $p=0.01$ ), with no significant changes in LVEDV between groups. There was a non-significant trend towards improvement in LV ejection fraction (Fig. 2).

\section{Native T1 Mapping}

Changes in native T1 values are shown in Table 3. There were significant reductions in global native $\mathrm{T} 1$ 
Table 1. Demographic and dialysis details

\begin{tabular}{|c|c|c|c|c|}
\hline & \multicolumn{2}{|c|}{ INHD group $(n=13)$} & \multicolumn{2}{|c|}{ CHD group $(n=12)$} \\
\hline Age, years & $55(35)$ & & $63(19)$ & \\
\hline Gender, male, $\%$ & $11(85)$ & & $10(83)$ & \\
\hline Dialysis vintage, months & $34.3 \pm 36.0$ & & $39.4 \pm 53.3$ & \\
\hline \multicolumn{5}{|l|}{ Cause of End stage renal disease, $n(\%)$} \\
\hline Diabetes mellitus & \multicolumn{2}{|l|}{$1(8)$} & \multicolumn{2}{|l|}{$1(8)$} \\
\hline Glomerulonephritis & \multicolumn{2}{|l|}{$4(30)$} & \multicolumn{2}{|l|}{$1(8)$} \\
\hline ADPKD & \multicolumn{2}{|l|}{$0(0)$} & \multicolumn{2}{|l|}{$1(8)$} \\
\hline Other & \multicolumn{2}{|l|}{$1(8)$} & \multicolumn{2}{|l|}{$4(34)$} \\
\hline Uncertain & \multicolumn{2}{|l|}{$7(54)$} & \multicolumn{2}{|l|}{$5(42)$} \\
\hline \multicolumn{5}{|l|}{ Vascular access, $n(\%)$} \\
\hline Arteriovenous fistula/graft & \multicolumn{2}{|l|}{$8(62)$} & \multicolumn{2}{|c|}{$12(100)$} \\
\hline Tunneled dialysis catheter & \multicolumn{2}{|l|}{$5(38)$} & \multicolumn{2}{|c|}{$0(0)$} \\
\hline \multicolumn{5}{|l|}{ Medical comorbidities, $n$} \\
\hline Diabetes mellitus & \multicolumn{2}{|l|}{2} & \multicolumn{2}{|l|}{2} \\
\hline Hypertension & \multicolumn{2}{|l|}{5} & \multicolumn{2}{|l|}{8} \\
\hline Ischaemic heart disease & \multicolumn{2}{|l|}{2} & \multicolumn{2}{|l|}{1} \\
\hline Atrial fibrillation & \multicolumn{2}{|l|}{2} & \multicolumn{2}{|l|}{0} \\
\hline Peripheral vascular disease & \multicolumn{2}{|l|}{1} & \multicolumn{2}{|l|}{0} \\
\hline Cerebrovascular disease & \multicolumn{2}{|l|}{0} & \multicolumn{2}{|l|}{1} \\
\hline Medications, $n$ & Baseline & Follow-up & Baseline & Follow-up \\
\hline ACE-i/ARB & 3 & 3 & 1 & 3 \\
\hline Beta-blocker & 3 & 3 & 5 & 3 \\
\hline Alpha blocker & 1 & 2 & 2 & 2 \\
\hline Calcium channel blocker & 1 & 2 & 3 & 2 \\
\hline Loop-diuretics & 1 & 1 & 2 & 1 \\
\hline Calcium containing phosphate binder & 4 & 3 & 4 & 3 \\
\hline Non-calcium containing phosphate binder & 5 & 3 & 3 & 3 \\
\hline
\end{tabular}

Table showing demographic data, including prescribed medications, and dialysis details. Values expressed are either: mean \pm SD or median (interquartile range). INHD, in-centre nocturnal haemodialysis; CHD, conventional haemodialysis; ADPKD, autosomal dominant polycystic kidney disease; ACE-i, angiotensin converting enzyme inhibitor; ARB, angiotensin II receptor antagonist.

(mean difference $[95 \%$ CIs] INHD $=-30.62 \mathrm{~ms}[-56.49$ to -4.75$], \mathrm{CHD}=0.4 \mathrm{~ms}$ [-25.47 to 26.27], $p=0.05)$ and non-septal native $\mathrm{T} 1$ values (mean difference [95\% CIs] INHD $=-30.93 \mathrm{~ms}[-58.44$ to -3.41$], \mathrm{CHD}=8.96 \mathrm{~ms}$ [ -18.56 to 36.47$], p=0.02$ ). Post hoc testing confirmed significant reductions in both global and non-septal native T1 time ( $p=0.02$ and $p=0.03$, respectively; Fig. 3a, $c)$. There was no significant change in septal native $\mathrm{T} 1$ values (Fig. 3b).

\section{Strain and $P W V$}

There were improvements in mean GCS and GLS in the INHD group between the start and the end of study $(-18.83 \pm 4.91,-20.15 \pm 5.09$ and $-15.51 \pm 4.35 \%$, $-17.69 \pm 2.66 \%$, respectively), though not statistically different with analysis of variance testing. Similar- ly mean PWV improved in the INHD group (8.67 \pm $4.17-7.59 \pm 3.08 \mathrm{~ms}$ ), though it was not significant (Table 3$)$.

\section{Relationships between Outcome Measures}

Relationships between changes in baseline and followup CMR outcome measures were evaluated (Fig. 4). Change in native $\mathrm{T} 1(\Delta \mathrm{T} 1 ;[r=0.526, p=0.03])$ and change in non-septal T1 $(\Delta$ nonseptalT1; $r=0.687, p=$ $0.01)$ correlated with change in LVM. There was no relationship found between $\triangle \mathrm{LVM}$ and change in septal T1 ( $\Delta$ septalT1; $r=0.145, p=0.59)$. No relationship was observed between $\Delta \mathrm{T} 1$ and change in hydration status assessed with bio-impedance spectroscopy ( $\Delta$ Hydration). Similarly, there were norelationships between $\Delta$ phosphate or $\Delta \mathrm{UF}$ rate and $\Delta \mathrm{T} 1$ or $\Delta \mathrm{LVM}$. 
Table 2. Changes in physical, humoral and quality-of-life outcomes between patients undergoing in-centre nocturnal vs. standard haemodialysis

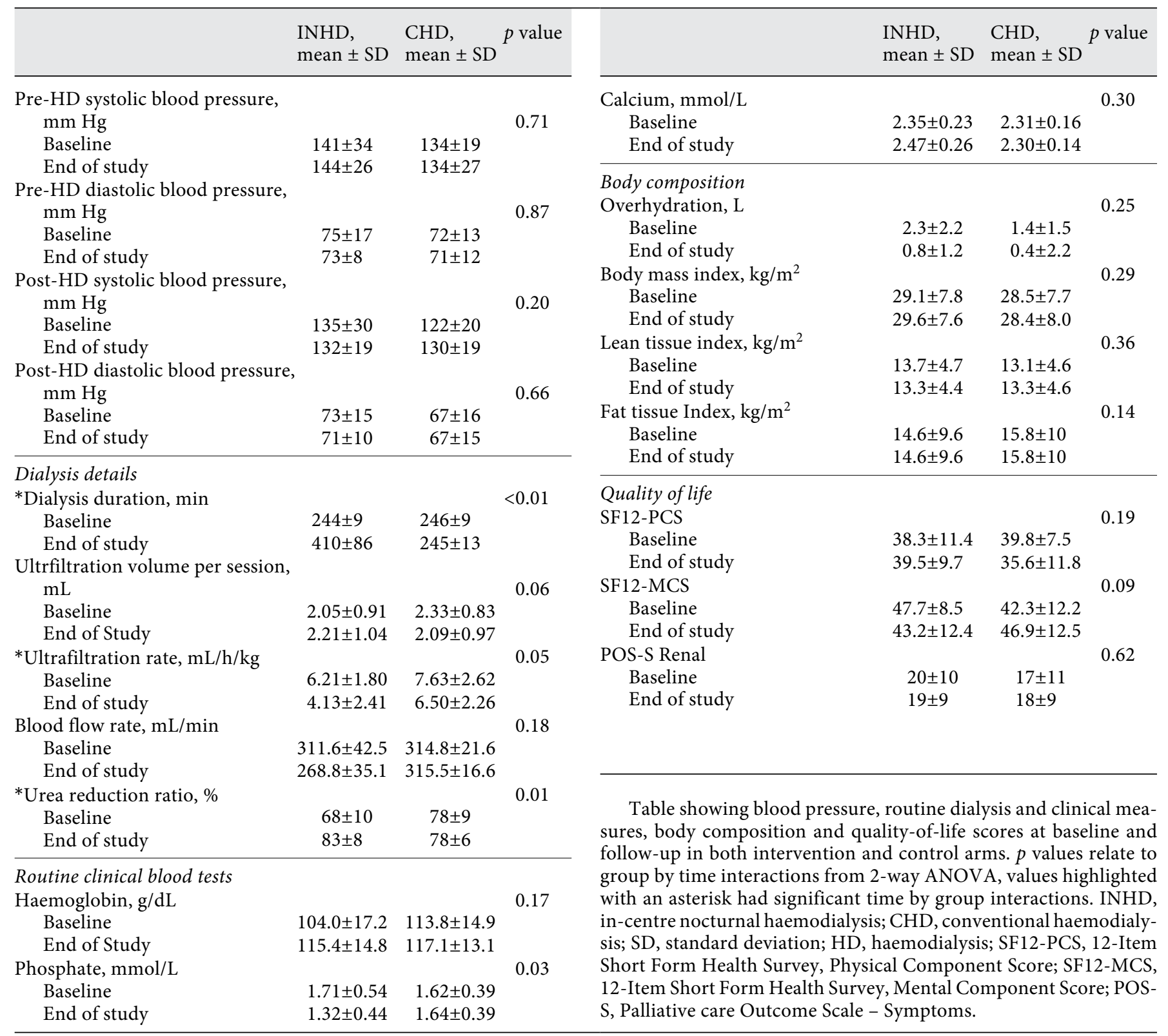

\section{Sensitivity Analyses}

Sensitivity analyses were conducted on CMR outcomes to account for missing data. We assumed no change for missing follow-up data and imputed baseline values [36]. This increased sample sizes to 11 in each group. Results for changes in LVM, LVM/LVEDV and non-septal T1 values remained statistically significant, while global T1 values showed a trend towards significance (mean difference [ $95 \% \mathrm{CIs}$ ] INHD $=17.58 \mathrm{~ms}$ [ -1.29 to 36.44$]$, $\mathrm{CHD}=$ $-0.29 \mathrm{~ms}$ [ -19.16 to 18.57$], p=0.12$ ).

Cardiac Remodelling and Nocturnal

Dialysis: The MIDNIGHT Study

\section{Discussion}

In-centre nocturnal HD was associated with a $12 \%$ reduction in LVM as previously described [22] with a final, overall change between INHD patients and controls of 22 g. This is comparable to trials of both frequent HD (6time per week) [13] and home nocturnal HD [37]. The observation that LVM and LVM/LVEDV reduced significantly compared to control patients, with no differences in LVEDV, confirms the reductions in LVM are unre- 
Table 3. Changes in cardiac structure, function and tissue characterization measured by cardiac MRI

\begin{tabular}{|c|c|c|c|c|c|c|c|}
\hline${ }^{*} \mathrm{LVM}, \mathrm{g}$ & & & \multirow{3}{*}{0.02} & Circumferential PSS, \% & & & \multirow{3}{*}{0.78} \\
\hline Baseline & $121 \pm 50$ & $95 \pm 20$ & & Baseline & $-18.83 \pm 4.91$ & $-20.62 \pm 3.29$ & \\
\hline End of study & $106 \pm 46$ & $102 \pm 27$ & & End of study & $-20.15 \pm 5.09$ & $-21.52 \pm 3.86$ & \\
\hline LVEDV, $\mathrm{mL}$ & & & 0.90 & Longitudinal PSS, \% & & & 0.32 \\
\hline${ }^{*} \mathrm{LVM} / \mathrm{LVEDV}, \mathrm{g} / \mathrm{mL}$ & & & \multirow{3}{*}{0.01} & Pulse wave velocity, ms & & & \multirow{3}{*}{0.21} \\
\hline Baseline & $0.67 \pm 0.11$ & $0.59 \pm 0.11$ & & Baseline & $8.67 \pm 4.17$ & $7.64 \pm 4.00$ & \\
\hline End of study & $0.60 \pm 0.09$ & $0.65 \pm 0.07$ & & End of study & $7.59 \pm 3.08$ & $8.13 \pm 3.11$ & \\
\hline \multicolumn{8}{|l|}{ LVEF, \% } \\
\hline Baseline & $50.5 \pm 7.5$ & $54.0 \pm 5.5$ & \multirow[t]{2}{*}{0.11} & & & & \\
\hline End of study & $54.2 \pm 6.6$ & $53.8 \pm 5.5$ & & \multirow{6}{*}{\multicolumn{4}{|c|}{$\begin{array}{l}\text { Table showing changes in left ventricular mass, volume, strain } \\
\text { and T1 values at baseline and follow-up in both intervention and } \\
\text { control arms. } p \text { values relate to group by time interactions from } \\
\text { 2-way ANOVA. Variables highlighted with an asterisk had signi- } \\
\text { ficant time by group interactions. MRI, magnetic resonance ima- } \\
\text { ging; INHD, in-centre nocturnal haemodialysis; CHD, conventio- } \\
\text { nal haemodialysis; LVM, left ventricular mass; LVEDV, left ven- } \\
\text { tricular end diastolic volume; LVEF, left ventricular ejection frac- } \\
\text { tion; PSS, peak systolic strain. }\end{array}$}} \\
\hline Baseline & $1,255 \pm 20$ & $1,247 \pm 47$ & \multirow[t]{2}{*}{0.02} & & & & \\
\hline End of study & $1,224 \pm 28$ & $1,256 \pm 33$ & & & & & \\
\hline \multicolumn{4}{|l|}{ Septal T1 value, ms } & & & & \\
\hline Baseline & $1,293 \pm 30$ & $1,300 \pm 44$ & \multirow[t]{2}{*}{0.37} & & & & \\
\hline End of study & $1,265 \pm 27$ & $1,289 \pm 40$ & & & & & \\
\hline
\end{tabular}

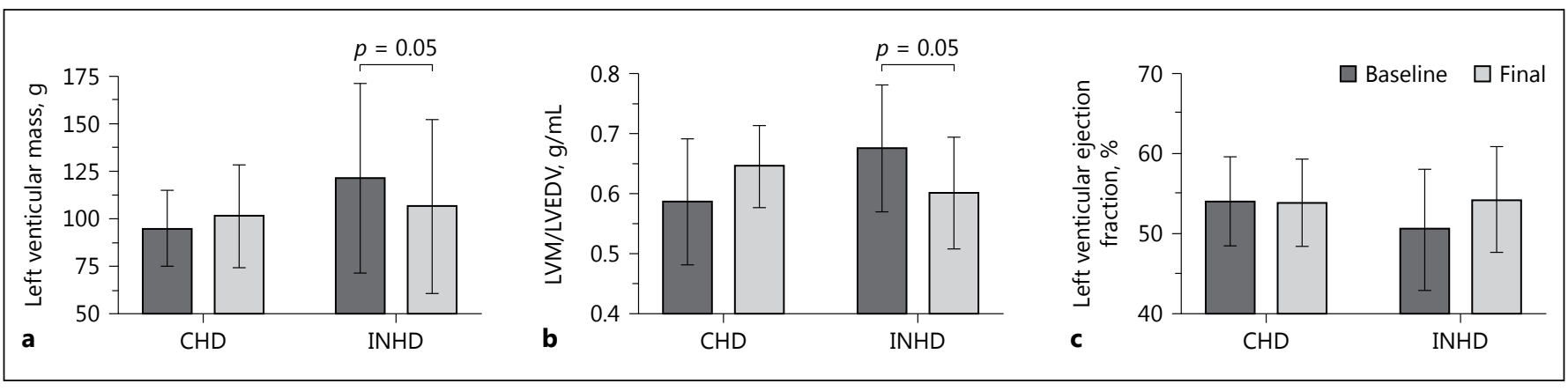

Fig. 2. Changes in left ventricular function and geometry. Data show changes in: (a) left ventricular mass; (b) LVM/LVEDV; and (c) left ventricular ejection fraction after 6-month for both CHD and INHD patients. Data shown are mean values with error bars representing standard deviation. CMR, cardiac magnetic resonance imaging; CHD, conventional haemodialysis; INHD, in-centre nocturnal haemodialysis; LVM, left ventricular mass; LVEDV, left ventricular end-diastolic volume. lated to cardiac loading status and suggests INHD promotes favourable cardiac remodelling. The reduction in native T1 mapping in the INHD group compared to controls suggests a reduction in myocardial fibrosis, with reductions seeming to be greatest in non-septal myocardium.

Our group has previously shown that one third of HD patients have increased native T1 signal in the interven- tricular septum that resemble areas of focal enhancement typically seen in patients who undergo gadolinium enhanced CMR [27, 38]. These areas have significantly higher native $\mathrm{T} 1 \mathrm{times}$ and are likely to represent replacement (irreversible) fibrosis. While native T1 times in non-septal myocardium of HD patients are higher than those in controls, there are fewer areas of discretely enhanced signal suggesting diffuse interstitial (reversible) 


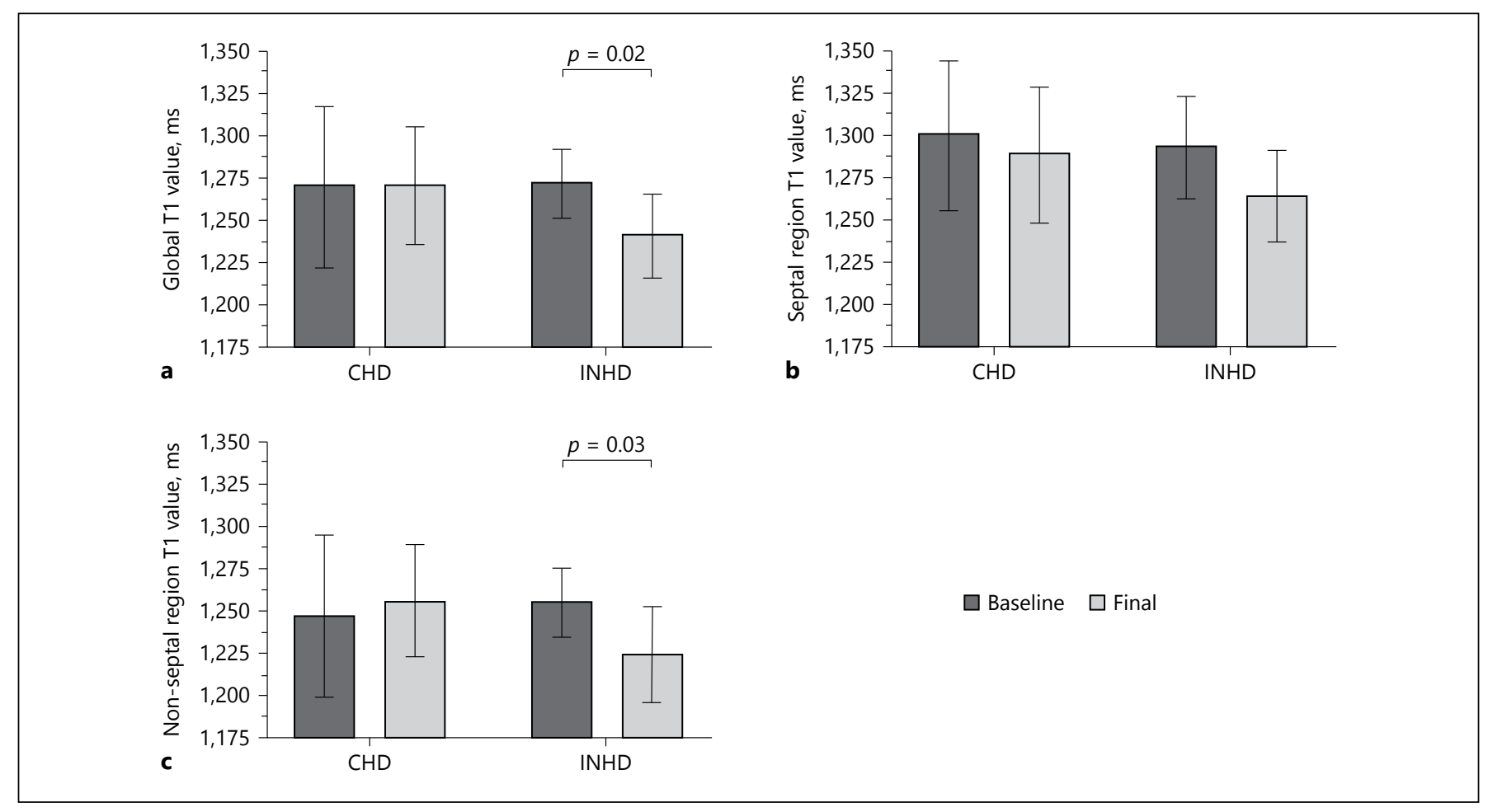

Fig. 3. Changes in global and regional $\mathrm{T} 1$ values of the left ventricle. Histograms show baseline and 6-month follow-up data for: (a) global T1 values; (b) septal region T1; and (c) non-septal region T1

fibrosis predominates in these areas. The observation in this study that native $\mathrm{T} 1$ reduced in the non-septal myocardium of INHD patients suggests reversal of diffuse interstitial myocardial fibrosis or myocardial inflammation. This hypothesis is supported by the relationships we have described between $\Delta \mathrm{LVM}, \Delta \mathrm{T} 1, \Delta$ nonseptalT1, and $\Delta$ septalT1. The $\triangle \mathrm{LVM}$ between scans correlated strongly with $\Delta \mathrm{T} 1$ and $\Delta$ nonseptalT1, but not $\Delta$ septalT1, suggesting reductions in LVM may be partly explained by reductions in non-septal diffuse interstitial fibrosis. These findings are supported by a study by Jin et al. [39] who showed that 12-month of INHD led to a reduction in LVM and myocardial fibrosis assessed with calibrated integrated backscatter derived from 2D echocardiography.

The observed change in LVM between the groups was driven by a combination of a reduction in LVM in the INHD (who had higher baseline LVM compared to controls) and an increase in LVM in the CHD group. While this may represent a "regression to the mean" phenomenon, the changes in LVM/LVEDV suggest that the changes are reflective of beneficial concentric remodelling. The reductions in native T1 related to INHD suggest an improvement in myocardial fibrosis. The trends towards values measure using cardiac magnetic resonance. Data shown are mean values with error bars representing SD. CHD, conventional haemodialysis; INHD, in-centre nocturnal haemodialysis. improvements in GCS, GLS and PWV are hypothesis generating and should be explored in larger studies of INHD, as modification of these measures may add mechanistic insight into the cardiac remodelling we have described.

The CMR outcome measures we have described are surrogate end points. The associations between LV hypertrophy, its progression and subsequent outcomes for HD patients are well known [40]. It is not clear, however, whether reduction in LVM is always associated with improved outcomes for HD patients; indeed a recent systematic review showed that there is no consistent association between LVM reduction and mortality [41]. This is possibly because LVM is an insufficiently sensitive measure of myocardial remodelling and cannot distinguish between interventions that lead to a reduction in myocyte hypertrophy and interventions that lead to a reduction in extra-cellular volume and interstitial fibrosis.

The observed improvement in phosphate control is an established benefit of extended HD times [16, 18, 22, 4244]. Additionally, the literature suggests that improvements in phosphate that accompany extended periods of $\mathrm{HD}$ are accompanied by a reduction in phosphate binders 

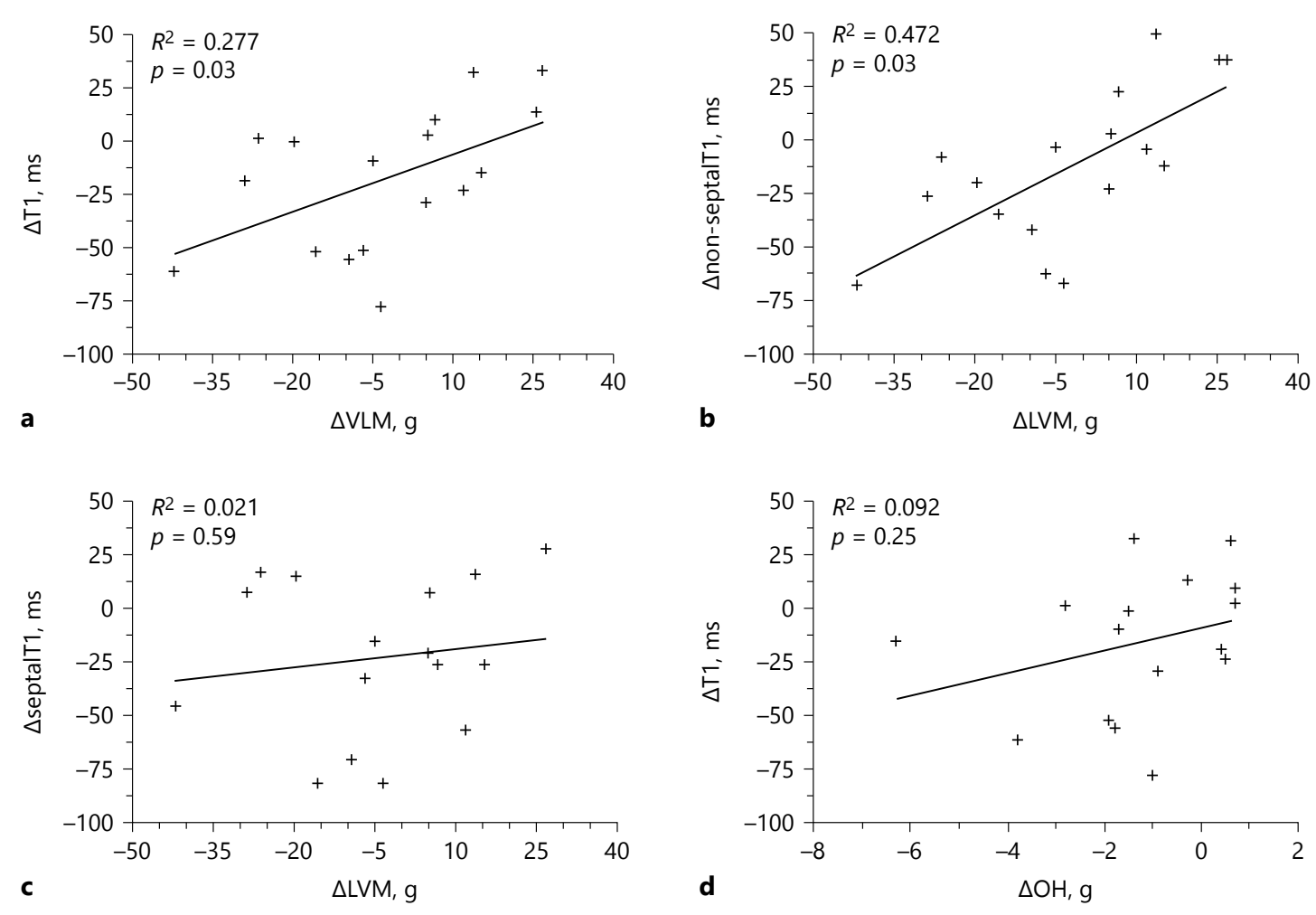

Fig. 4. The relationships between changes in baseline and followup CMR outcome measures. Plots showing the correlations between: (a) Change in left ventricular (LV) mass and change in T1 score; (b) Change in left ventricular (LV) mass and change in non- septal T1 score; (c) Change in left ventricular (LV) mass and change in septal T1 score; and (d) change in over-hydration status and change in T1 score. CMR, cardiac magnetic resonance imaging; LVM, left ventricular mass; $\mathrm{OH}$, over hydration.
[22, 45-47]. Although we did not demonstrate a significant reduction in the number of phosphate binders, this is likely due to the small numbers of patients in this study and the limited follow-up. Similarly, while we have not demonstrated any association between change in phosphate and change in LVM or native T1, the reduction in serum phosphate is striking. Given the associations between levels of serum phosphate and FGF-23-driven LVH [48-50], the links between phosphate and the improvement in cardiac remodelling we describe should be tested in larger studies of extended HD. Similarly, INHD was not associated with significant differences in body composition compared to CHD, including pre-dialysis over-hydration. We did not show any significant change in systolic or diastolic blood pressure or in the use of anti-hypertensives as shown previously $[18,21]$. Some have suggested that this may be one of the reasons for reductions in LVM associated with INHD. The fact that we have seen a reduction in LVM with no change in blood pressure may suggest that factors independent of blood pressure may contribute to the improvements in cardiac remodelling we describe. However, it is also possible that our small sample size masks important effects of blood pressure on INHD-related cardiac remodelling. As we have shown previously, while there is a small increase in the total ultrafiltration volumes of patients undergoing INHD compared to CHD, this is abrogated by the increased length of time over which patients dialyse. The absolute ultrafiltration rates we report are significantly lower in the INHD group than those in the CHD group. Speed of ultrafiltration associates strongly with frequency and severity of intra-dialytic myocardial stunning events [51], and repeated intra-dialytic myocardial stunning is known to lead to myocardial fibrosis [6]. The reduction in ultrafiltration rates and consequent reduction in myocardial stunning events is a possible mechanism for the reduction in native $\mathrm{T} 1$ times and myocardial fibrosis that we have shown in this study in patients undergoing 
INHD. As previously described, INHD programs are an alternative way of offering extended dialysis sessions to patients and increasing the choice available to both patients and clinicians $[16,22]$. INHD programs may lead to improved quality-of-life scores compared to CHD [18], and a reduction in dialysis-related symptoms and sleep quality $[47,52]$.

\section{Limitations}

The sample size is small, but this was designed as a feasibility study. The design of this study was non-randomized with patients electing to switch to INHD introducing intrinsic selection bias. Those in the CHD group were older than those in the INHD group (non-significant) but also had lower baseline values for LVM. Native T1 mapping is a promising imaging biomarker for myocardial fibrosis in HD patients, but there are no histological studies in HD patients that confirm the relationship between histological myocardial fibrosis and native $\mathrm{T} 1$ value.

From the total number of patients recruited to the INHD and CHD group, $22 \%$ did not complete all aspects of the study. Although this loss of patients appears high, this does not represent the feasibility of the intervention or the integrity of the data after sensitivity analysis. Within the INHD arm of the study, all patients continued with INHD unless a superior form of renal replacement therapy was offered (transplantation $n=2$ ). Furthermore, an INHD programme has been established across the Leicester Renal Network since 2014 and provides 10\% of in- centre dialysis capacity. Thus, INHD is an acceptable method of renal replacement therapy and we do not anticipate recruitment to INHD to be a limiting factor.

\section{Conclusions}

A 6-month program of extended hours INHD is associated with favourable LV remodelling and reduction in myocardial T1 (a surrogate of myocardial fibrosis) compared to control patients on conventional HD. This is the first study to report the effects of an intervention on native $\mathrm{T} 1$ values in $\mathrm{HD}$ patients, and that native $\mathrm{T} 1$ is modifiable. This promising data should be viewed as hypothesis generating and require testing in adequately powered studies.

\section{Acknowledgements}

This study is independent research arising from a grant from the Van Geest Foundation. This work is supported by the National Institute for Health Research Leicester Biomedical Research Centre. The views expressed in this publication are those of the authors and not necessarily those of the National Health Service, the National Institute for Health Research or the Department of Health.

\section{Disclosure Statement}

None of the authors have any competing interests to declare, financial or otherwise.

\section{References}

1 Foley RN, Parfrey PS, Sarnak MJ: Epidemiology of cardiovascular disease in chronic renal disease. J Am Soc Nephrol 1998;9:S16S23.

2 US Renal Data System USRDS 2013 Annual Data Report: Atlas of chronic Kidney Disease and End-Stage Renal Disease in the United States, 2013 .

3 Amann K, Rychlik I, Miltenberger-Milteny $\mathrm{G}$, et al: Left ventricular hypertrophy in renal failure. Kidney Int Suppl 1998;54:S78S85.

4 Amann K, Buzello M, Simonaviciene A, et al: Capillary/myocyte mismatch in the heart in renal failure-a role for erythropoietin? Nephrol Dial Transplant 2000;15:964-969.

5 Harnett JD, Foley RN, Kent GM, et al: Congestive heart failure in dialysis patients: prevalence, incidence, prognosis and risk factors. Kidney Int 1995;47:884-890.
6 Burton JO, Jefferies HJ, Selby NM, et al: Hemodialysis-induced repetitive myocardial injury results in global and segmental reduction in systolic cardiac function. Clin J Am Soc Nephrol 2009;4:1925-1931.

7 Chiu DY, Sinha S, Kalra PA, et al: Sudden cardiac death in haemodialysis patients: preventative options. Nephrology (Carlton) 2014;19: 740-749.

8 Herzog CA, Mangrum JM, Passman R: Sudden cardiac death and dialysis patients. Semin Dial 2008;21:300-307.

9 Herzog CA, Strief JW, Collins AJ, et al: Causespecific mortality of dialysis patients after coronary revascularization: why don't dialysis patients have better survival after coronary intervention? Nephrol Dial Transplant 2008; 23:2629-2633.

10 McIntyre CW, Burton JO, Selby NM, et al: Hemodialysis-induced cardiac dysfunction is associated with an acute reduction in global and segmental myocardial blood flow. Clin J Am Soc Nephrol 2008;3:19-26.

11 Burton JO, Jefferies HJ, Selby NM, et al: Hemodialysis-induced cardiac injury: determinants and associated outcomes. Clin J Am Soc Nephrol 2009;4:914-20.

12 Burton JO, Korsheed S, Grundy BJ, et al: Hemodialysis-induced left ventricular dysfunction is associated with an increase in ventricular arrhythmias. Ren Fail 2008;30:701709.

13 FHN Trial Group, Chertow GM, Levin NW, et al: In-center hemodialysis six times per week versus three times per week. N Engl J Med 2010;363:2287-2300.

14 Lacson E Jr, Lazarus M. Dialysis time: does it matter? A reappraisal of existing literature. Curr Opin Nephrol Hypertens 2011;20:189194. 
15 Lacson E Jr, Wang W, Lester K, et al. Outcomes associated with in-center nocturnal hemodialysis from a large multicenter program. Clin J Am Soc Nephrol 2010;5:220-226.

16 Graham-Brown MP, Churchward DR, Smith AC, et al: A 4-month programme of in-centre nocturnal haemodialysis was associated with improvements in patient outcomes. Clin Kidney J 2015;8:789-795.

17 Barraclough N, Mooney D, Mullins K, et al: Improved cardiac structure and function in a patient transitioned to in-centre nocturnal haemodialysis. Nephrology 2012;17:94.

18 Ok E, Duman S, Asci G, et al: Comparison of 4- and 8-h dialysis sessions in thrice-weekly in-centre haemodialysis: a prospective, casecontrolled study. Nephrol Dial Transplant 2011;26:1287-1296.

19 Jin X, Rong S, Mei C, et al: Effects of thriceweekly in-center nocturnal vs. conventional hemodialysis on integrated backscatter of myocardial tissue. Hemodial Int 2011;15: 200-210.

20 Maduell F, Arias M, Duran CE, et al: Nocturnal, every-other-day, online haemodiafiltration: an effective therapeutic alternative. Nephrol Dial Transplant 2012;27:16191631.

21 David S, Kuempers P, Eisenbach GM, et al: Prospective evaluation of an in-centre conversion from conventional haemodialysis to an intensified nocturnal strategy. Nephrol Dial Transplant 2009;24:2232-2240.

22 Wald R, Goldstein MB, Perl J, et al: The association between conversion to in-centre nocturnal hemodialysis and left ventricular mass regression in patients with end-stage renal disease. Can J Cardiol 2016;32:369-377.

23 Nesrallah GE, Lindsay RM, Cuerden MS, et al: Intensive hemodialysis associates with improved survival compared with conventional hemodialysis. J Am Soc Nephrol 2012;23: 696-705.

24 Hunold P, Vogt FM, Heemann UW, et al: Myocardial mass and volume measurement of hypertrophic left ventricles by MRI-study in dialysis patients examined before and after dialysis. J Cardiovasc Magn Reson 2003;5: 553-561.

25 Graham-Brown MP, Rutherford E, Levelt E, et al: Native T1 mapping: inter-study, interobserver and inter-center reproducibility in hemodialysis patients. J Cardiovasc Magn Reson 2017;19:21.

26 Edwards NC, Moody WE, Chue CD, et al: Defining the natural history of uremic cardiomyopathy in chronic kidney disease: the role of cardiovascular magnetic resonance. JACC Cardiovasc Imaging 2014;7:703-714.
27 Graham-Brown MP, March DS, Churchward DR, et al: Novel cardiac nuclear magnetic resonance method for noninvasive assessment of myocardial fibrosis in hemodialysis patients. Kidney Int 2016;90:835-844.

28 Rutherford E, Talle MA, Mangion K, et al: Defining myocardial tissue abnormalities in end-stage renal failure with cardiac magnetic resonance imaging using native T1 mapping. Kidney Int 2016;90:845-852.

29 Graham-Brown MP, Burton JO, McCann GP: The use of T1 mapping to define myocardial fibrosis in haemodialysis patients. Eur Heart J Cardiovasc Imaging 2016;17:832.

30 Churchward DR, Graham-Brown MP, Preston $\mathrm{R}$, et al: Investigating the effects of 6 months extended duration, in-centre nocturnal versus conventional haemodialysis treatment: a non-randomised, controlled feasibility study. BMJ Open 2016;6:e012583.

31 Mactier R, Hoenich N, Breen C: Renal association clinical practice guidelines haemodialysis. Nephron Clin Pract 2011;118(suppl 1): c241-c86.

32 Singh A, Ford I, Greenwood JP, et al: Rationale and design of the PRognostic Importance of MIcrovascular Dysfunction in asymptomatic patients with Aortic Stenosis (PRIMIDAS): a multicentre observational study with blinded investigations. BMJ Open 2013;3: e004348.

33 Kramer CM, Barkhausen J, Flamm SD, et al: Standardized cardiovascular magnetic resonance (CMR) protocols 2013 update. J Cardiovasc Magn Reson 2013;15:1.

34 Singh A, Steadman CD, Khan JN, et al: Intertechnique agreement and interstudy reproducibility of strain and diastolic strain rate at 1.5 and 3 tesla: a comparison of feature-tracking and tagging in patients with aortic stenosis. J Magn Reson Imaging 2015;41:11291137.

35 Odudu A, Eldehni MT, McCann GP, et al: Characterisation of cardiomyopathy by cardiac and aortic magnetic resonance in patients new to hemodialysis. Eur Radiol 2015: $1-13$.

36 Thabane L, Mbuagbaw L, Zhang S, et al: A tutorial on sensitivity analyses in clinical trials: the what, why, when and how. BMC Med Res Methodol 2013;13:92.

37 Culleton BF, Walsh M, Klarenbach SW, et al: Effect of frequent nocturnal hemodialysis vs conventional hemodialysis on left ventricular mass and quality of life: a randomized controlled trial. JAMA 2007;298:1291129.

38 Mark P, Johnston N, Groenning B, et al: Redefinition of uremic cardiomyopathy by con- trast-enhanced cardiac magnetic resonance imaging. Kidney Int 2006;69:1839-1845.

39 Jin X, Rong S, Mei C, et al: Effects of in-center nocturnal versus conventional hemodialysis on endothelial dysfunction. Ther Apher Dial 2012;16:334-340.

40 Foley RN, Parfrey PS, Harnett JD, et al: The prognostic importance of left ventricular geometry in uremic cardiomyopathy. J Am Soc Nephrol 1995;5:2024-2031.

41 Badve SV, Palmer SC, Strippoli GF, et al: The Validity of left ventricular mass as a surrogate end point for all-cause and cardiovascular mortality outcomes in people With CKD: a systematic review and meta-analysis. Am J Kidney Dis 2016;68:554-563.

42 Eloot S, Van Biesen W, Dhondt A, et al: Impact of hemodialysis duration on the removal of uremic retention solutes. Kidney Int 2008; 73:765-770.

43 Chan C, Floras J, Miller J, et al: Improvement in ejection fraction by nocturnal haemodialysis in end-stage renal failure patients with coexisting heart failure. Nephrology Dialysis Transplantation 2002;17:1518-1521.

44 Rocco MV: More frequent hemodialysis: back to the future? Adv Chronic Kidney Dis 2007; 14:e1-e9.

45 Powell JR, Oluwaseun O, Woo YM, et al: Ten years experience of in-center thrice weekly long overnight hemodialysis. Clin J Am Soc Nephrol 2009;4:1097-1101.

46 Alloatti S, Molino A, Manes M, et al: Long nocturnal dialysis. Blood Purif 2002;20:525530.

47 Bugeja A, Dacouris N, Thomas A, et al: Incenter nocturnal hemodialysis: another option in the management of chronic kidney disease. Clin J Am Soc Nephrol 2009;4:778-783.

48 Faul C, Amaral AP, Oskouei B, et al: FGF23 induces left ventricular hypertrophy. J Clin Invest 2011;121:4393-4408.

49 Chue CD, Edwards NC, Moody WE, et al: Serum phosphate is associated with left ventricular mass in patients with chronic kidney disease: a cardiac magnetic resonance study. Heart 2012;98:219-224.

50 Chue CD, Townend JN, Moody WE, et al: Cardiovascular effects of sevelamer in stage 3 CKD. J Am Soc Nephrol 2013;24:842-852.

51 Jefferies HJ, Virk B, Schiller B, et al: Frequent hemodialysis schedules are associated with reduced levels of dialysis-induced cardiac injury (myocardial stunning). Clin J Am Soc Nephrol 2011;6:1326-1332.

52 van Eps C, Hawley C, Jeffries J, et al: Changes in serum prolactin, sex hormones and thyroid function with alternate nightly nocturnal home haemodialysis. Nephrology 2012;17:42-47. 